\title{
Surgical treatment of primary hyperparathyroidism due to parathyroid tumor: A 15-year experience
}

\author{
LU FENG, XU ZHANG and SHAN-TING LIU \\ Department of Head and Neck Surgery, Affiliated Cancer Hospital of Zhengzhou \\ University, Zhengzhou, Henan 450008, P.R. China
}

Received December 14, 2014; Accepted February 1, 2016

DOI: $10.3892 / \mathrm{ol} .2016 .4905$

\begin{abstract}
The aim of this study was to highlight our experience over a 15-year period in dealing with primary hyperparathyroidism (PHPT) due to a parathyroid tumor. Parathyroidectomy is the standard therapy for patients with PHPT. Our study included all patients with PHPT treated by parathyroidectomy at the Affiliated Cancer Hospital of Zhengzhou University, China. Between 1998 and 2013, a total of 107 patients were recruited. Their clinical data, presentation, laboratory examinations, imageological diagnoses and surgical approaches were analyzed retrospectively. Eighty-four cases (78.5\%) were followed up. During a median follow-up period of 5.7 years, a total of 80 patients were without recurrence and metastasis. The main symptoms of PHPT patients were palpable neck mass, joint pains and pathological fracture. The high levels of preoperative parathyroid hormone $(\mathrm{PTH})$ and serum calcium in PHPT patients decreased to below the normal upper limit within 3 days of surgery. The sensitivity of neck ultrasonography, sestamibi scanning, CT, MRI and the combination of three or four types of test were $86.0 \%, 90.4 \%, 80.8 \%, 79.6 \%$ and $96.1 \%$, respectively. A $50 \%$ or greater drop in PTH levels within 20 min compared with the highest PTH levels before surgery occurred in 95/107 cases (88.8\%). Transient hypocalcemia was the most common surgical complication. The ultrasonography and sestamibi scan is the most effective examination for parathyroid tumor. The 20 min PTH measurement appears to be extremely useful, and avoids unnecessary bilateral exploration.
\end{abstract}

Correspondence to: Professor Shan-Ting Liu, Department of Head and Neck Surgery, Affiliated Cancer Hospital of Zhengzhou University, 127 Dongming Road, Zhengzhou, Henan 450008, P.R. China

E-mail: liushanting@163.com

Abbreviations: PHPT, primary hyperparathyroidism; PTH, parathyroid hormone; CT, computed tomography; MRI, magnetic resonance imaging; IOPTH, intraoperative parathyroid hormone

Key words: primary hyperparathyroidism, parathyroidectomy, parathyroid, parathyroid hormone, intra-operative parathyroid hormone

\section{Introduction}

Primary hyperparathyroidism (PHPT) is characterized by hypercalcemia resulting from the overproduction of parathyroid hormone (PTH) by one or multiple hyperfunctioning parathyroid glands. It is the third most common endocrine disorder with a reported incidence of $\sim 0.25 \%$ in the general population (1). The incidence of PHPT increases with age, and it occurs more frequently in females than in males (2). Research has confirmed that the majority of patients with PHPT require surgery due to the risk of renal stones, osteoporosis, cardiovascular disease, and in certain cases silent complications of renal impairment (3). The majority of PHPT cases are caused by a single parathyroid adenoma (75-85\% of cases) (4). In 1928, Dr. Isaac Olch (5) performed the first successful parathyroidectomy at Barnes Hospital in St. Louis, MO, USA, and currently this operation remains one of the most effective treatments, providing great potential for cure in patients with PHPT. As the clinical features of PHPT are variable and lack specificity, there is no clear consensus with regard to the most effective treatment of patients with the condition. Therapeutic strategies generally arise through clinical experiences. In this study, we report our experience of dealing with 107 cases of PHPT due to parathyroid tumor over the past 15 years, as well as our experience in treatment of the condition with parathyroidectomy.

\section{Materials and methods}

Patient data. In this retrospective study, we recruited 107 patients who were operated on for PHPT in our surgical department between January 1998 and December 2013. Of the 107 patients, there were 80 females and 27 males, with a median age of 57 years (range, 28-79 years). The disease duration ranged from 6 months to 15 years (median, 25 months). Clinical information was derived from a thorough review of the medical records and the institutional patient database. Follow-up data were obtained through correspondence and outpatient department visits, with the average follow-up period being 5.7 years (range, 1-15 years).

Presentation and diagnosis. Forty-three patients exhibited an asymptomatic neck mass. Metabolic bone disease was radiologically observed in 37 cases. Nineteen patients were 
Table I. Sensitivity and positive predictive values for various preoperative diagnostic modalities.

\begin{tabular}{lccccc}
\hline Index & $\begin{array}{c}\text { Ultrasound } \\
(\%)\end{array}$ & $\begin{array}{c}\text { Sestamibi } \\
(\%)\end{array}$ & $\begin{array}{c}\text { CT } \\
(\%)\end{array}$ & $\begin{array}{c}\text { MRI } \\
(\%)\end{array}$ & $\begin{array}{c}\text { Association of ultrasound } \\
\text { and sestamibi (\%) }\end{array}$ \\
\hline Sensitivity & 86.0 & 90.4 & 80.8 & 79.6 & 94.7 \\
Positive predictive value & 95.3 & 94.1 & 83.6 & 85.0 & 100.0 \\
\hline
\end{tabular}

CT, computed tomography; MRI, magnetic resonance imaging.

asymptomatic despite hypercalcemia. Eighteen were initially observed to have constitutional symptoms including palpitations, nausea, fatigue, weight loss and memory deficit. Twelve patients exhibited lithangiuria but had normal renal function. Several patients suffered more than one symptom.

To localize hyperfunctioning parathyroid glands, a variety of imaging techniques were used, including neck ultrasonography, Tc-99 m sestamibi scanning, computed tomography (CT) and magnetic resonance imaging (MRI). All patients underwent neck ultrasonography. The Tc-99 m sestamibi scan was performed in 83 cases (77.6\%). In addition, 47 patients accepted CT or MRI scanning.

Treatment. Under general anesthesia, all our cases underwent a focused parathyroidectomy associated with a rapid intraoperative PTH (IOPTH) assay monitoring. PTH levels were measured prior to parathyroid tumor excision, and subsequent post-excision measurements followed at 10 and $20 \mathrm{~min}$, if a sufficient reduction in PTH value was not observed. A $50 \%$ or greater drop in PTH values from the pre-incision or pre-excision level was considered to indicate a surgical success.

Ethics. Ethical approval was granted from the Zhengzhou University Ethical Review Board.

\section{Results}

Pathological presentation. A total of 104 patients (97.2\%) had benign lesions, and 3 patients (2.8\%) had parathyroid carcinoma. Of the 104 patients, there were 97 cases (90.7\%) of single parathyroid adenoma, 4 cases $(3.7 \%)$ of multiple parathyroid adenoma or combined parathyroid hyperplasia, and 3 cases (2.8\%) of parathyroid hyperplasia. Eighty-four cases $(78.5 \%)$ were followed up. During the follow-up period, 2 patients (2.4\%) suffered parathyroid adenoma recurrence, 2 patients (2.4\%) succumbed to parathyroid carcinoma, 1 case of carcinoma survived for 34 months before succumbing to postoperative lung and bone metastasis, and 1 succumbed to a cardiovascular accident 21 months after surgery. The remaining patients did not experience recurrence or metastasis.

Sensitivity and positive predictive values. Ultrasonographic examination of the neck revealed 92 cases of occupied lesions in the parathyroid gland and 10 cases in the thyroid gland (Fig. 1). The remaining 5 cases had normal neck ultrasonography results. The sensitivity and positive predictive values were $86.0 \%$ and $95.3 \%$, respectively. Eighty-three patients underwent Tc-99 m sestamibi scanning. The accumulation

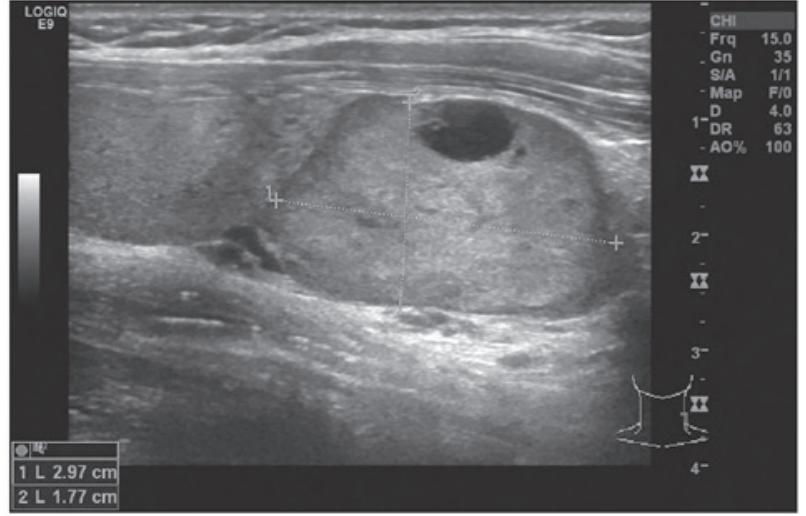

Figure 1. Ultrasound image of a parathyroid adenoma.

of radioactivity was observed in 75 of these patients (Fig. 2). The sensitivity and positive predictive values were $90.4 \%$ and $94.1 \%$. The combination of an ultrasound and $99 \mathrm{~m} \mathrm{Tc}$-sestamibi scan localized hyperfunctioning parathyroid in 76/82 patients (92.7\%). The sensitivity in identifying parathyroid tumors by CT and MRI was $80.8 \%$ and $79.6 \%$, respectively (Table I).

PTH levels. Prior to surgery, elevated PTH levels were observed in all patients. The preoperative serum calcium was abnormally elevated in 86 patients (80.4\%). The levels of serum calcium and PTH in patients with parathyroid hyperplasia and carcinoma were significantly higher than those in parathyroid adenoma patients (Table II).

A 50\% or greater drop in PTH levels within $10 \mathrm{~min}$ compared with the highest PTH levels before surgery occurred in 86 patients $(80.4 \%)$. In 9 cases the $>50 \%$ drop in PTH levels was attained after $20 \mathrm{~min}(8.4 \%)$. A $>50 \%$ drop in PTH levels within 20 min was achieved by $95 / 107$ patients (88.8\%; Table III).

Complications. Surgical complications were observed in nine patients: three had wound infections, two had hematoma necessitating re-operation and two had transient recurrent laryngeal nerve paresis. Transient hypocalcemia occurred in 19 cases (17.8\%). Symptoms ranged from face, hand and foot numbness to limb twitching. All of these patients were treated with calcium and vitamin D per os and intravenously with resolution within 1 month. There were no cases of permanent hypocalcemia.

Statistical analysis. Results were recorded and are expressed as the means \pm standard deviation. Statistical significance 
Table II. Comparison of observed indices among various pathological parathyroid lesions.

\begin{tabular}{|c|c|c|c|}
\hline Index & $\begin{array}{c}\text { Parathyroid } \\
\text { adenoma }(n=101)\end{array}$ & $\begin{array}{c}\text { Parathyroid } \\
\text { hyperplasia }(n=3)\end{array}$ & $\begin{array}{c}\text { Parathyroid } \\
\text { carcinoma }(n=3)\end{array}$ \\
\hline PTH before surgery (pmol/l, mean \pm SD) & $121.3 \pm 29.0$ & $143.0 \pm 76.3^{\mathrm{a}}$ & $188.2 \pm 56.5^{\mathrm{b}}$ \\
\hline Serum calcium before surgery $(\mathrm{mmol} / \mathrm{l}$, mean $\pm \mathrm{SD})$ & $2.9 \pm 0.4$ & $3.3 \pm 0.6^{\mathrm{c}}$ & $4.3 \pm 1.9^{\mathrm{d}}$ \\
\hline
\end{tabular}

${ }^{\mathrm{a}} \mathrm{P}=0.004 ;{ }^{\mathrm{b}} \mathrm{P}=0.001 ;{ }^{\mathrm{c}} \mathrm{P}=0.005 ;{ }^{\mathrm{P}} \mathrm{P}=0.000$, compared with parathyroid adenoma. $\mathrm{PTH}$, primary hyperparathyroidism; $\mathrm{SD}$, standard deviation.

Table III. Decrease in PTH following surgery.

\begin{tabular}{lcc} 
Decrease in PTH & $\begin{array}{c}>50 \% \text { decrease in PTH } \\
\text { within } 10 \text { min }\end{array}$ & $\begin{array}{c}>50 \% \text { decrease in } \\
\text { PTH after } 20 \text { min }\end{array}$ \\
\hline Patients & 86.0 & 9.0 \\
$\%$ & 80.4 & 8.4 \\
\hline
\end{tabular}

PTH, primary hyperparathyroidism.

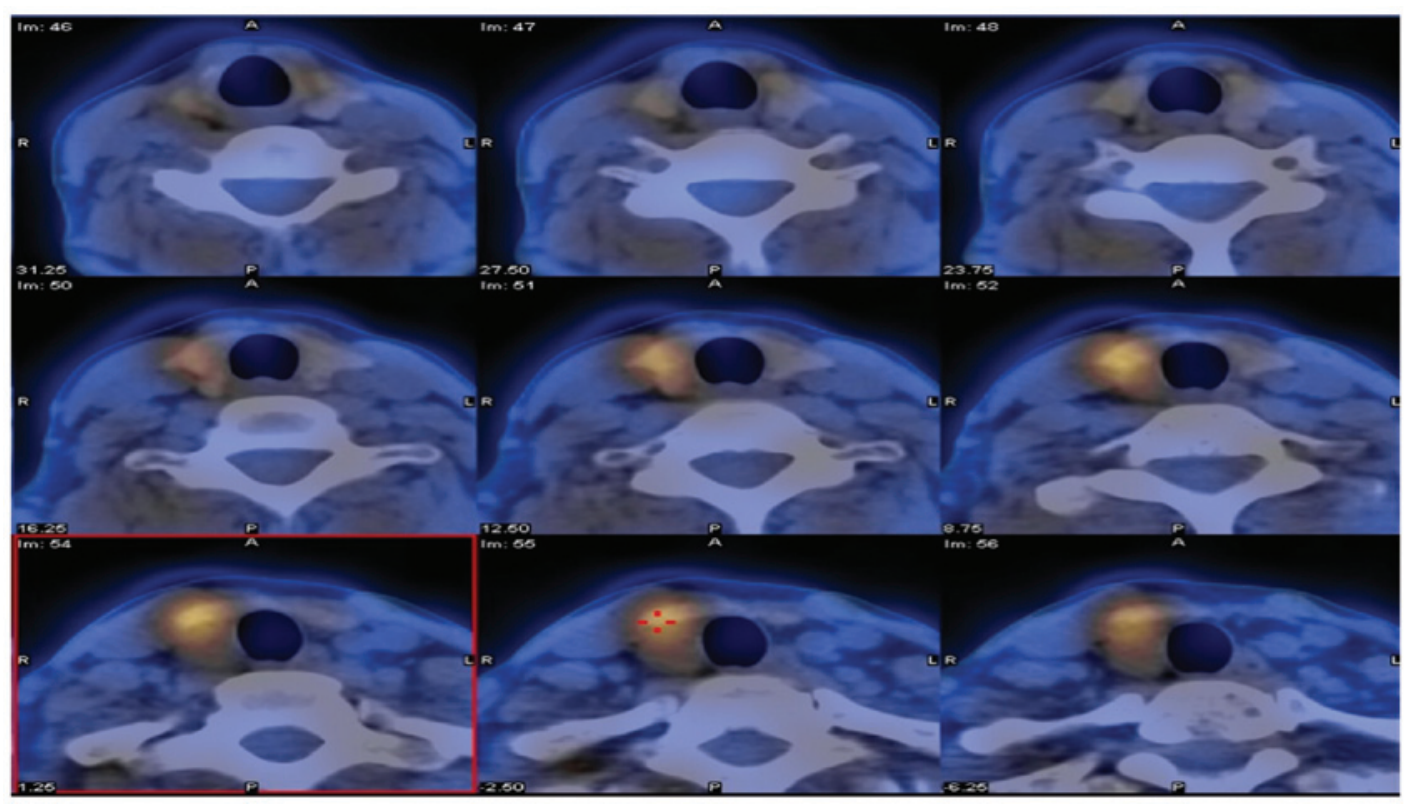

Figure 2. Strong accumulation of Tc-99 $\mathrm{m}$ in the right thyroidal lobe was observed.

was determined using the Chi-square test. All analyses were performed using the Statistical Package for Social Science (SPSS) version 16.0 (SPSS Inc., Chicago, IL, USA). $\mathrm{P}<0.05$ was considered to indicate a statistically significant difference.

\section{Discussion}

Ruda et al (6) reviewed of the diagnosis and treatment of PHPT from 1995 to 2003 and concluded that single parathyroid adenoma accounts for $\sim 90 \%$ of benign tumors of the parathyroid. Our study revealed a similar result: $90.7 \%$ of our cases had single-gland disease.

The diagnosis of PHPT is generally made biochemically following the measurement of elevated serum calcium and
PTH levels $(1,4)$.In this group, we did not observe any significant differences between the parathyroid hyperplasia and the parathyroid carcinoma groups with regard to the PTH and serum calcium values before surgery. However, the levels of serum calcium and PTH in patients with parathyroid hyperplasia and carcinoma were significantly higher than those of parathyroid adenoma patients. However, the small sample size of three cases means that the study should be replicated with a larger sample.

There is wide variation in the anatomic location of the parathyroid glands, and in certain patients hyperfunctioning glands are hard to locate during surgery. In these cases, reliable preoperative localization of abnormal parathyroid glands has become invaluable. The two most common imaging 
modalities are sestamibi scanning and neck ultrasonography. High-resolution ultrasound is a effective examination to locate enlarged parathyroid glands in the neck. One of the advanced features of neck ultrasound is the ability to study the thyroid gland concurrently for any abnormalities. In addition, neck ultrasound is also beneficial as it is inexpensive, it does not use ionizing radiation, and it has high sensitivity (7). The sensitivity of ultrasonography for the localization of abnormal parathyroid glands generally varies in the literature from $61 \%$ to $85 \%$ (8-11). However, in the present study, we noted a higher sensitivity of $86.0 \%$. The variation in these numbers could be due to the fact that ultrasonography is operator-dependent. At our institution, ultrasonography is performed by a professional neck ultrasound doctor, and these individuals are likely to have the best understanding of neck anatomy. Much like ultrasonography, sestamibi also has a high sensitivity for the detection of abnormal parathyroid glands, and is particularly useful in detecting small, posterior adenomas (12). According to our statistical results, when ultrasound and sestamibi scans are used together, the sensitivity of preoperative localization of parathyroid adenomas increases. These findings are consistent with the majority of studies. The reported sensitivity ranges between $94 \%$ and $99 \%$ (13). CT and MRI are less used but are useful in patients with failed parathyroidectomy or persistent PHPT to identify ectopic glands (8).

In their study on the reassessment of PTH monitoring, Gawande et al (14) noted that it is less necessary to monitor the value of IOPTH when there are concordant imaging studies. Conversely, other studies (15) advocate the use of IOPTH monitoring to guide parathyroid excision in all patients with PHPT.

Sokoll (16) argued that using IOPTH in conjunction with reliable preoperative localization facilitates the ability to perform minimally invasive surgery in an ambulatory surgery setting with improved success intraoperatively, increased patient satisfaction postoperatively, and decreased costs perioperatively. This argument has been a hot debate in the literature in recent years and is likely to continue to be a point of discussion in the foreseeable future. However, from our point of view, surgical treatment without IOPTH assay may be appropriate in certain institutions where financial or access constraints prevent the use of IOPTH assay, and at this point, how to correctly locate the abnormal parathyroid glands is particularly significant.

At our institution, IOPTH assay is still widely used. In particular, blood collection timing appears to be a critical step (17). The widely used Miami criterion (rapid IOPTH value drop $>50 \%$ from the highest levels either pre-incision or at 10 min after gland excision) is reported to have a high overall accuracy. However, other authors have reported error rates as high as $16 \%$ due to false-negative and false-positive results (18). They suggested that $20 \mathrm{~min}$ of PTH monitoring should be performed (19). In this study, we performed IOPTH monitoring after $10 \mathrm{~min}$ if a $>50 \%$ drop of PTH levels was not observed. In nine patients $(6.4 \%)$, a $>50 \%$ decrease of PTH was observed only after $20 \mathrm{~min}$, and an unnecessary bilateral exploration was thus avoided.

Cure of PHPT may be defined as normocalcemia 6 months after surgery, irrespective of the level of PTH (20).
According to Hessman et al, the success rate of parathyroidectomy is greater than $95 \%$, with low complication rates in cases performed by experienced surgeons (21). The results of the present study are similar to those of Hessman et al (21).

Parathyroid carcinoma is an extremely rare malignancy and is reported to occur in less than $0.1 \%$ to $5 \%$ of patients with PHPT (22-24). The same result was demonstrated in the current study. There were 3 cases of parathyroid carcinoma (2.8\%) among the 107 cases of PHPT due to parathyroid tumor in our study. To date, the diagnostic criteria for parathyroid carcinoma has been inconsistent. Parathyroid carcinoma may therefore be under- or overdiagnosed. In our experience, the obscure boundary or/and invasion of adjacent tissues is the key indicator of parathyroid carcinoma. For parathyroid carcinoma, males and females are equally affected, usually in the fourth or fifth decade of life. Short of a major biological or molecular breakthrough, surgery remains the most effective therapeutic and palliative option (25).

Considering the small number of parathyroid carcinoma cases, our clinical diagnostic and treatment experience is limited. Further research is required on this topic to confirm our findings.

In conclusion, the symptoms of PHPT vary and lack specificity. Parathyroidectomy provides the most effective treatment for PHPT due to parathyroid tumor. The ultrasonography and sestamibi scan is the most effective examination for parathyroid tumor. The $20 \mathrm{~min}$ PTH measurement appears to be extremely useful, and avoids unnecessary bilateral exploration and the associated risk of complications with only a slight increase in the duration of surgery and the cost.

\section{References}

1. Bilezikian JP and Silverberg SJ: Clinical practice. Asymptomatic primary hyperparathyroidism. N Engl J Med 350: 1746-1751, 2004.

2. Udén P, Chan A, Duh QY, Siperstein A and Clark OH: Primary hyperparathyroidism in younger and older patients: Symptoms and outcome of surgery. World J Surg 16: 791-797; discussion 798, 1992.

3. Philips IJ, Kurzawinski TR and Honour JW: Potential pitfalls in intraoperative parathyroid hormone measurements during parathyroid surgery. Ann Clin Biochem 42: 453-458, 2005.

4. Fraser WD: Hyperparathyroidism. Lancet 374: 145-158, 2009.

5. Meyer A, Brabant G and Behrend M: Surgical treatment of primary hyperparathyroidism. Eur J Med Res 29: 287-291, 2005.

6. Ruda JM, Hollenbeak CS and Stack BC Jr: A systematic review of the diagnosis and treatment of primary hyperparathyroidism from 1995 to 2003. Otolaryngol Head Neck Surg 132: 359-372, 2005.

7. Felger EA and Kandil E: Primary hyperparathyroidism. Otolaryngol Clin North Am 43: 417-432, 2010.

8. Johnson NA, Tublin ME and Ogilvie JB: Parathyroid imaging: technique and role in the preoperative evaluation of primary hyperparathyroidism. AJR Am J Roentgenol 188: 1706-1715, 2007.

9. Meilstrup JW: Ultrasound examination of the parathyroid glands. Otolaryngol Clin North Am 37: 763-778; ix, 2004.

10. Solorzano CC, Carneiro-Pla DM and Irvin GL III: Surgeon-performed ultrasonography as the initial and only localizing study in sporadic primary hyperparathyroidism. J Am Coll Surg 202: 18-24, 2006.

11. Ghaheri BA, Koslin DB, Wood AH and Cohen JI: Preoperative ultrasound is worthwhile for reoperative parathyroid surgery. Laryngoscope 114: 2168-2171, 2004.

12. Augustine MM, Bravo PE and Zeiger MA: Surgical treatment of primary hyperparathyroidism. Endocr Pract 17 (Suppl 1): S75-S82, 2011. 
13. Haber RS, Kim CK and Inabnet WB: Ultrasonography for preoperative localization of enlarged parathyroid glands in primary hyperparathyroidism: comparison with $(99 \mathrm{~m})$ technetium sestamibi scintigraphy. Clin Endocrinol (Ozf) 57: 241-249, 2002.

14. Gawande AA, Monchik JM, Abbruzzese TA, Iannuccilli JD, Ibrahim SI and Moore FD Jr: Reassessment of parathyroid hormone monitoring during parathyroidectomy for primary hyperparathyroidism after 2 preoperative localization studies. Arch Surg 141: 381-384; discussion 384, 2006.

15. Hanif F, Coffey JC, Romics L Jr, O'Sullivan K, Aftab F and Redmond HP: Rapid intraoperative parathyroid hormone assay more than just a comfort measure. World J Surg 30: 156-161, 2006.

16. Sokoll LJ: Measurement of parathyroid hormone and application of parathyroid hormone in intraoperative monitoring. Clin Lab Med 24: 199-216, 2004

17. Di Stasio E, Carrozza C, Pio Lombardi C, Raffaelli M, Traini E, Bellantone R and Zuppi C: Parathyroidectomy monitored by intra-operative PTH: the relevance of the $20 \mathrm{~min}$ end-point. Clin Biochem 40: 595-603, 2007.

18. Calò PG, Pisano G, Tatti A, Medas F, Boi F, Mariotti S and Nicolosi A: Intraoperative parathyroid hormone assay during focused parathyroidectomy for primary hyperparathyroidism: Is it really mandatory? Minerva Chir 67: 337-342, 2012.
19. Lombardi CP, Raffaelli M, Traini E, Di Stasio E, Carrozza C, De Crea C, Zuppi C and Bellantone R: Intraoperative PTH monitoring during parathyroidectomy: the need for stricter criteria to detect multiglandular disease. Langenbecks Arch Surg 393: 639-645, 2008.

20. Suliburk JW and Perrier ND: Primary hyperparathyroidism. Oncologist 12: 644-653, 2007.

21. Hessman O, Stålberg P, Sundin A, Garske U, Rudberg C, Eriksson LG, Hellman P and Akerström G: High success rate of parathyroid reoperation may be achieved with improved localization diagnosis. World J Surg 32: 774-781; discussion 782-783, 2008.

22. Obara T and Fujimoto Y: Diagnosis and treatment of patients with parathyroid carcinoma: an update and review. World J Surg 15: 738-744, 1991.

23. Favia G, Lumachi F, Polistina F and D'Amico DF: Parathyroid carcinoma: sixteen new cases and suggestions for correct management. World J Surg 22: 1225-1230, 1998

24. Rodgers SE and Perrier ND: Parathyroid carcinoma. Curr Opin Oncol 18: 16-22, 2006.

25. Givi B and Shah JP: Parathyroid carcinoma. Clin Oncol (R Coll Radiol) 22: 498-507, 2010. 